\title{
Philonsorbonne
}

9 | 2015

Année 2014-2015

\section{Doctorales 2014}

Résumés des interventions des quatrièmes Doctorales de Philosophie (2014)

\section{(2) OpenEdition}

\section{Journals}

Édition électronique

URL : https://journals.openedition.org/philonsorbonne/746

DOI : $10.4000 /$ philonsorbonne.746

ISSN : 2270-7336

Éditeur

Publications de la Sorbonne

\section{Édition imprimée}

Date de publication : 1 janvier 2015

Pagination : 89-134

ISSN : 1255-183X

Référence électronique

«Doctorales 2014 », Philonsorbonne [En ligne], 9 | 2015, mis en ligne le 11 janvier 2015, consulté le 08 juin 2021. URL : http://journals.openedition.org/philonsorbonne/746 ; DOI : https://doi.org/10.4000/ philonsorbonne.746 


\section{Doctorales 2014 *}

Sur la personne et ses modes chez Pierre Abélard

Ángela Beatriz ÁvALOS

Sur la possibilité d'une conception active et pluraliste de l'expérience tactile

Alireza BANI SADR

Le monde à perte de vue :

l'admiration de la montagne chez Pétrarque et Gesner

Thibault BARRIER

Chiralité et réflexion : approche du schématisme transcendantal

Eric BEAURON

Énigmes et paraboles. Langage et expression «corporelle » des prophètes dans le Traité théologico-politique de Spinoza

Nicolas BOUTELOUP

Le platonisme pur-sang de Mark Balaguer

Costanza BREVINI

La maladie dans ses effets, un écart à la norme physiologique

ou bien une nouvelle norme pour vivre?

Jacques CABARET

Pour quelles raisons cherche-t-on une définition de la vie?

Quel genre de mot est « vie »?

Sébastien DUTREUIL

Sur l'objet mathématique et la réalité mathématique

dans le cadre du structuralisme non éliminanif : le débat Shapiro-Parsons

Edgar Fernando GÁLVEZ

L'idéalité comme condition de possibilité de la connaissance en général

et d'une métaphysique légitime

Eylem HACIMURATOĞLU

Les méthodes de Claude Lancelot, entre pédagogie et philosophie du langage à Port Royal

Max HARDT

Un traité de médecine est-il une illustration métaphysique?

L'exemple de l'amour chez Avicenne

Anne-Sophie JOUANNEAU

Anthropocène : histoire, nature et modernité

Pierre de JOUVANCOURT

(*). Dans cette rubrique sont publiés les résumés des interventions ayant eu lieu lors des quatrièmes Doctorales de Philosophie organisées à la Sorbonne, les 05, 06 et 07 juin 2014, par les doctorants Olivier D'Jeranian (PhiCo), Hélène Garello (CHSPM), Quentin Hardy (CETCOPRA), Michal TARASIEWICZ (GRAMATA) et David ZAREBSKI (IHPST). 
La preuve de la sphéricité de l'univers (Timée 33b 1-7; De caelo II 4)

Daniel LAROSE

Merleau-Ponty et les deux voies d'un réalisme anthropologisé

Manuel LEVAL-DuCHÉ

Le langage dans la méthode résolutive compositive : de Galilée à Hobbes

Charles NKOURISSA

De la prévention à la prédiction :

la médecine est-elle parvenue à une nouvelle connaissance de l'individu?

Delphine OLIVIER

Deux morales sociales - Bergson et Durkheim

Kotaro ONO

Image et signe dans la troisième Critique

Elena PARTENE

Un constitutionnalisme démocratique est-il possible?

Juliette Roussin

Les figures de l'oubli chez Nietzsche

Arnaud SOROSINA

L'objection «nationale » de David Miller

aux théories cosmopolitiques sur la justice mondiale

Mathilde UNGER 


\section{Ángela Beatriz Ávalos}

Personne et Personnalité dans la philosophie de Pierre Abélard Thèse dirigée par Christophe Grellard abasoto@gmail.com

\section{Sur la personne et ses modes chez Pierre Abelard}

Cet article a pour but de présenter quelques aspects fondamentaux de la pensée d'Abélard à travers la notion de personne.

À la fois propre et impropre à son époque, la philosophie abélardienne est essentiellement critique (voire analytique), et si celle-ci gagne à être comprise de manière imbriquée, ce n'est cependant pas sous la forme d'une « unité » ou d'un « système » qu'elle doit être conçue. C'est sous ce même mode d'approche que nous chercherons à penser la personne dans ses différentes acceptions, telle qu'elle est présentée dans la Theologia Christiana de Pierre Abélard :

Tribus itaque seu quatuor modis ac pluribus fortassis hoc nomen " persona » sumitur, aliter uidelicet a theologis, aliter a grammaticis, aliter a rhetoricis uel in comoediis, ut supra determinatum est ${ }^{1}$.

Le mot "personne », inséré principalement dans un cadre théologique (à la différence de la notion d'individu, qui se rencontre exclusivement dans un cadre logique), est conçu comme un outil ou un moyen conceptuel pour expliquer l'unité et La Trinité divine. Cette notion signifie, cependant, différemment (aliter, aliter, aliter) pour le théologien, le grammairien, le

1. «La personne peut être prise de trois manières différentes, suivant qu'elle est considérée par des théologiens, des grammairiens, des rhéteurs, et il y a aussi une personne pour le comédien » P. Abélard, Theologia Christiana (TC), 3.181. 
rhéteur et le comédien: les personnes trinitaires, les «je», «tu », « il » grammaticaux et ce que l'on pourrait nommer l'apparence (cette troisième forme sera rapportée au rhéteur et au comédien en essayant d'expliciter leur lien).

Quel est le statut de la personne pour chacun de ces personnages, ces notions sont-elles liées ? La notion de personne est-elle une figure vide, ou a-t-elle quelque chose d'établi a priori ? Voilà les interrogations qui animent ce texte.

Le sujet de la personne devient un objet tant de la théologie que des arts du langage (trivium); ou plutôt - nous essayons de le montrer - la question de la personne devient un des axes fondateurs de la philosophie d'Abélard en tant que théologie dans le langage.

Le trivium est au service de la théologie, de la compréhension du discours sur Dieu, car la parole divine, nous étant donnée sous la forme du langage écrit (c'est-à-dire d'ordre humain et fini) doit être analysée et comprise - elle doit être lue et intelligée - comme telle, en acceptant les limites que cela suppose. L'analyse de la personne traverse ainsi des colonnes structurelles de la philosophie abélardienne, telles que: la signification, la nomination, l'utilité des arts du langage et l'humanité de l'expérience théologique comme expérience herméneutique.

Ce travail s'articule donc en quatre parties :

- le fondement de la philosophie abélardienne, ou bien le lien entre dialectique et théologie comme condition de possibilité pour penser la notion de personne ;

- les personnes trinitaires ou personnes théologiques et leur rapport intime (et nécessaire) au langage ;

- la personne grammaticale comme fondement d'une interpersonnalité et « abstraction»;

- la rhétorique et sa personne en tant qu'acteur et outil pour l'affection du récepteur par le discours. 
Alireza BANI SADR

La détection de l'objet de l'expérience perceptive

Thèse dirigée par Maximilian Kistler

alirezabanisadr@gmail.com

\section{Sur la possibilité d'une conception active et pluraliste de l'expérience tactile}

S'il existe une interdépendance entre action et perception, le sens du toucher en est l'exemple paradigmatique. Pourtant, l'individuation même du sens de toucher a fait l'objet de controverses dans la philosophie de la perception depuis Aristote : d'une part, l'appareil sensoriel du toucher inclut des récepteurs variés remplissant des fonctions spécifiques, et d'autre part, les propriétés dites tangibles semblent être diverses et parfois hétérogènes. L'individuation du toucher par critère unique tel que l'organe sensoriel, le stimulus physique, l'objet propre/le contenu représentationnel et le caractère phénoménal, nous conduit souvent à l'hétérogénéité. Toutefois, pour résoudre ce problème certaines propositions ont été formulées, dont les suivantes :

1. Les théories corporelles. Ces approches essaient principalement de réduire d'une part les propriétés tangibles multiples à une classe fondamentale des propriétés, les propriétés spatiales, et d'autre part d'établir que le fait d'être conscient de notre corps nous donne accès aux propriétés spatiales des objets externes ${ }^{2}$.

2. La théorie de la pression. Adoptant une approche intentionnelle à l'égard de l'individuation, les tenants de cette théorie considèrent la pression et la tension comme objet propre et primaire du toucher. Comme l'indiquent

2. D. M. Armstrong, Bodily sensations, Routledge \& Kegan Paul, 1962. 
ses défenseurs, cette thèse conduit à admettre que seules la pression et la tension constituent les propriétés perçues à travers le toucher. D'autres propriétés, comme la texture, la vibration et le poids, sont perçues indirectement et en vertu de la perception de la pression et de la tension ${ }^{3}$.

3. La théorie pluri-sensorielle $d u$ toucher. Le toucher présente des propriétés distinctes et parfois hétérogènes telles que la texture, la vibration, le poids, la température, qui seront assignées au même objet tangible via le toucher ${ }^{4}$. Ce qui distingue l'expérience tactile des autres expériences, c'est le liage des propriétés (feature binding) dans le toucher qui consiste à assigner des qualités phénoménales aux objets tangibles à travers des actions exploratoires.

Notre étude porte principalement sur trois questions: le toucher comprend-il des systèmes sensoriels et des qualités phénoménales multiples ? Le toucher est-il une modalité perceptive active ou autrement dit, y a-t-il une interdépendance constitutive entre action et perception dans le toucher? Y a-t-il un lien entre l'«activité » et la «multiplicité » du toucher? Nous essayons d'analyser les difficultés auxquelles se heurtent les deux premières approches vis-à-vis du toucher mentionnées plus haut. Nous nous interrogeons également sur les défis que doit relever la troisième approche, l'approche pluri-sensorielle ou «pluraliste». Nous montrons pourquoi le questionnement sur la perception tactile doit être déplacé de la recherche de l'objet propre du toucher à la question du liage des propriétés tangibles. Nous établissons, par la suite, les conditions suivant lesquelles une conception pluraliste du toucher - c'est-à-dire celle qui reconnaîtrait le toucher en tant qu'il comporte une multiplicité de systèmes sensoriels et de propriétés sensibles - serait possible. Enfin, nous reconsidérons ces conditions dans le cas de l'expérience tactile sous l'angle de son rapport à l'action.

3. O. Massin, L'objectivité du toucher, Métaphysique et Perception, $\mathrm{PhD}$ thesis, Aix-Marseille, 2010. - O. Massin et F. de Vignemont, "Touch », in The Oxford Handbook of the Philosophy of Perception, M. Matthen (dir.), Oxford University Press, 2013.

4. M. Fulkerson, «The unity of haptic touch», Philosophical Psychology, 24(4), (2011), p. 493-516. 
Thibault BARRIER

Le théâtre de l'admiration: surprise et nouveauté aux XVI et XVII siècles

Thèse dirigée par Chantal Jaquet thbarrier@gmail.com

\section{Le monde à perte de vue : l'admiration de la montagne chez Pétrarque et Gesner}

Depuis les pages que Burkhardt lui a consacrées en 1860 dans $L a$ Civilisation de la Renaissance en Italie, la lettre de Pétrarque sur L'Ascension du mont Ventoux (1353) passe pour le texte inaugural de la modernité, dans lequel l'homme moyenâgeux détournait son regard du « livre », pour enfin contempler directement, par une vue d'en haut, la nature dans toute sa diversité. Pétrarque, le premier, aurait entrepris l'ascension d'un sommet "mû par le seul désir de voir » les choses terrestres, et aurait ainsi établi l'horizon désormais immanent du regard humain. Or, il nous semble au contraire que Pétrarque reste sur le seuil de la modernité, sans s'y engager toutefois. De l'Histoire romaine de Tite-Live, qui lui fournit le motif de son ascension, aux Confessions d'Augustin, qui lui révèlent la profonde vanité de l'admiration des cimes, l'itinéraire de Pétrarque est saturé de réminiscences biographiques et littéraires. Bien plus qu'une expérience visuelle, c'est un exercice mémoriel qui fonde le récit de la lettre. Ce n'est pas le spectacle d'une nature ordonnée qui se donne à voir, mais les tourments d'une âme désirante qui se donnent à entendre. L'ascension de Pétrarque ne montre rien, elle invite au contraire au retour à soi, en fermant les yeux sur le charme sensible du monde extérieur. Devant le panorama des sommets, ce n'est pas seulement la vue, mais l'homme tout entier qui se perd. 
Or, s'il faut que le passage à la modernité se repère au plaisir pris à la vue directe et surplombante de la nature, nous en trouvons un témoignage beaucoup plus décisif deux siècles plus tard, dans deux lettres du naturaliste zurichois Conrad Gesner, sur L'Admiration de la montagne (1541), et sur la Description du Fracmont (1555). Dans la première, l'admiration du spectacle des montagnes n'est plus appréhendée à travers le prisme d'une âme inquiète de succomber à la libido spectandi, elle est au contraire revendiquée comme l'aptitude d'un esprit disponible à la grandeur et à la diversité de la nature. Gesner inverse le topos du locus horribilis: tous les éléments qui suscitaient au préalable de l'effroi (monts abrupts, escarpements inaccessibles, épaisses forêts) sont désormais les objets d'un intense plaisir sensible. Vu d'en haut, le monde ordinaire devient méconnaissable, il apparaît comme un monde renouvelé au sein duquel la vue jouit de se perdre.

Dans la seconde lettre, le motif de l'ascension est, comme pour Pétrarque, fourni par le désir de vérifier des fables anciennes concernant un lac de montagne aux propriétés climatiques étranges depuis que Ponce Pilate s'y serait noyé. Si le lexique du merveilleux est encore convoqué, ce n'est plus pour qualifier le caractère incompréhensible de puissances naturelles occultes, mais pour rendre compte de l'inépuisable variété des phénomènes naturels qui déborde toujours les anticipations de la perception. L'admiration constitue alors l'affect adéquat pour se rapporter à cette infinie nouveauté du monde dont la montagne offre l'abrégé. 
Eric BEAURON

Analogie, affinité, système : Kant et la vocation architectonique de la connaissance Thèse dirigée par Christian Bonnet eric.beauron@gmail.com

\section{Chiralité et réflexion : approche du schématisme transcendantal}

Dans son opuscule intitulé $D u$ premier fondement de la différence des régions de l'espace (1768), Kant mentionne pour la première fois le paradoxe des objets symétriques non congruents: "J'appelle corps non congruent à un autre, un corps qui est tout à fait égal et semblable à celui-ci, sans toutefois pouvoir être enfermé dans les mêmes limites ${ }^{5} »$; il s'agit de penser la " possibilité d'espaces tout à fait semblables et égaux, et cependant non congruents ${ }^{6} \gg$.

Il y a cependant lieu de montrer que, en vertu de ses propres conditions d'intelligibilité, la chiralité ne définit pas un paradoxe spatial, lié à l'intuition externe et à la géométrie, mais un paradoxe de la réflexion transcendantale, qui concerne donc au premier chef les rapports entre entendement et intuition lorsqu'ils se rapportent l'un à l'autre dans la structure du schématisme. La chiralité permet ainsi d'éclairer l'opération de subsomption qui est la clef de voûte de la déduction transcendantale dans l'« Analytique des principes" de la Critique de la raison pure. La subsomption peut s'opérer parce qu'entendement et intuition sont comme la main gauche et la main droite : foncièrement inversées l'une par rapport à l'autre, ces deux

5. Kant, Du premier fondement de la différence des régions de l'espace, in Quelques opuscules pré-critiques, trad. S. Zac, Paris, Vrin, 1970, p. 96.

6. Ibid., p. 97. 
facultés peuvent néanmoins s'épouser, s'homogénéiser dans une même forme commune, le schème, comme le font nos deux mains en se pressant l'une contre l'autre. En ramenant la chiralité à sa fonction transcendantale, on montre donc, non seulement, que le kantisme a bien des mains - et qu'elles ne sont pas aussi pures que ne le suggérait Péguy dans sa fameuse boutade - mais surtout, on est en mesure de repenser l'articulation entre entendement et intuition en prenant pour fil conducteur le thème de l'inversion $d u$ sens qui est interne à la réflexion transcendantale. On vérifie la puissance opératoire de ce régime transcendantal de la chiralité en montrant qu'elle permet de redessiner le schématisme de la relation dans les Analogies de l'expérience, où les catégories de la substance, de la causalité et de la communauté ne sont pas, ainsi que le voudrait Kant, dans un rapport exclusif et univoque avec leurs modes temporels respectifs (permanence, succession, simultanéité). Ce que l'entendement pense à titre de règles intellectuelles de la synthèse en vue l'expérience possible ne dessine que l'image inversée, en miroir, des données immédiates de l'intuition. Celles-ci ne peuvent être subsumées que dans la mesure où l'entendement les comprend, mais à l'envers. En termes kantiens, la natura formaliter spectata est le symétrique non congruent de la natura materialiter spectata. 
La grammaire du vrai : modèle mathématique, modèle littéraire et modèle pictural chez Spinoza

Thèse dirigée par Chantal Jaquet nicolas.bouteloup@gmail.com

\section{Énigmes et paraboles. \\ Langage et expression « corporelle » des prophètes dans le Traité théologico-politique de Spinoza}

L'objectif de cet article est d'étudier la figure du prophète dans les premiers chapitres du Traité théologico-politique, afin d'en tirer des acquis quant à une certaine forme d'expression positive ne répondant pourtant pas à la rigueur du modèle géométrique. Pour ce faire, l'objectif a été d'analyser en détail l'expression du prophète, d'une part en tant qu'il est un discours imaginatif, et d'autre part en tant qu'il est un discours corporel. Spinoza caractérise en effet les prophètes par leur puissance imaginative et leur vertu, et non pas comme chez Maïmonide par une quelconque supériorité rationnelle. Pourtant, en réduisant la portée rationnelle des prophètes, et donc par là même, de leurs discours et enseignements, Spinoza donne une positivité à cette expression imaginative qui est la leur. Contrairement au discours rationnel qui se borne rigoureusement à dire le vrai, le discours imaginatif peut composer une infinité d'idées, qu'elles soient vraies ou fausses. À l'opposé d'un type de discours rationnel et rigoureux, mais limité et abstrait, le discours prophétique se veut illimité et «corporel» (et donc ancré dans l'expérience affective), mais confus et imaginaire. L'imagination, au travers des paraboles et des énigmes, n'est pas qu'une manière de percevoir, mais aussi une manière de délivrer la révélation et "d'enseigner », qui dépasse la simple oralité : si l'expression du prophète est «corporelle et imaginative», c'est aussi en tant que tout son corps 
est mobilisé pour transmettre ses idées, tout comme chez l'orateur qui ne fait pas que déclamer, mais qui accompagne toujours son discours d'une gestuelle, d'un engagement du corps dans son ensemble.

$\mathrm{Si}$ la figure du prophète ainsi que son mode d'expression nous paraissent essentiels, c'est parce qu'ils donnent de manière plus générale des indications sur un traitement possible de la fiction au sein de la doctrine spinoziste. Bien que la fiction soit, du point de vue de la connaissance, rejetée comme fausse dans le Traité de la réforme de l'entendement, elle peut pourtant être le lieu d'enseignements éthiques. Le langage fictionnel est avant tout ce mode d'expression imaginaire qui mélange, associe des idées, tisse des liens invisibles entre des objets que la raison ne pourrait cautionner, mais qui pourtant peuvent participer à un enseignement du vrai. Passer par un discours fictionnel, formuler un mythe ou bien lire un roman, ça n'est pas «mentir», se «tromper», ni énoncer des choses fausses (ça ne le serait qu'à partir du moment où l'on se place justement dans une analyse épistémologique), mais cela peut être au contraire une manière de créer un réseau de sens, libre, susceptible de variations et de modulations au travers de multiples interprétations. Cette analyse cherche donc à pointer la positivité d'un discours prophétique et fictionnel chez Spinoza, ce qui permettra par la suite de retrouver ce type de discours au sein même de la méthode géométrique ou, devrait-on dire, dans les interstices de cette dernière. 
Costanza BREVINI

Platonisme Procedurelle

Thèse dirigée par Marco Panza

costanza.brevini@unimi.it

\section{Le platonisme pur-sang de Mark Balaguer}

Le philosophe américain Mark Balaguer est auteur d'une version du platonisme non classique en mathématiques, appelé FBP (full-blooded Platonism) ou platonisme pur-sang. Balaguer est concerné à répondre au dilemme de Benacerraf, célèbre argument sur l'impossibilité d'obtenir la connaissance des objets mathématiques au sens platonicien. Le problème est que si les objets mathématiques sont abstraits, éternels et indépendants de l'existence d'un esprit qui les pense, qui sont comme le platonicien les décrit, alors la question se pose de savoir comment accéder à ces objets pour en obtenir connaissance.

La stratégie de Mark Balaguer est de montrer que le platonisme en mathématiques peut être compatible avec une épistémologie naturaliste. Il le fait en trouvant un moyen d'expliquer comment les hommes peuvent obtenir la connaissance des objets mathématiques, abstraits, éternels, indépendants.

Balaguer part d'un examen emprunté par l'argument du village népalais de Hartry Field : si existent tous les villages népalais possibles, alors il est possible de connaître ces villages comme ils sont faits et ce qui s'y passe, même sans aucune relation ou contact avec ces villages. Autrement dit, s'il y a tous les villages népalais possibles, il est suffisant de rêver ou inventer un village népalais pour avoir connaissance de quelque chose qui existe, mais avec qui on n'a aucun contact. De même, Balaguer fait valoir que toutes les théories mathématiques pures et cohérentes décrivent correctement une collection d'objets abstraits, parce qu'il y a tous les objets mathématiques 
qui sont logiquement possibles (c'est-à-dire sans aboutir à l'absurde). Sur le versant mathématique, ce genre de platonisme fournit une bonne interprétation philosophique de la liberté extrême qui caractérise la pratique des mathématiques, explique l'existence de questions indécidables et ouvertes en mathématiques et légitime les moyens de justification pragmatique.

Dans la relation que j'ai présentée, je me demandais si le platonisme pur-sang du Balaguer est également fort du point de vue philosophique, surtout s'il est vraiment capable de répondre à l'argument épistémologique contre le platonisme de Benacerraf (1973). Je suis arrivée à la conclusion que le platonisme pur-sang du Mark Balaguer propose un account philosophique des mathématiques qui concilie les pratiques mathématiques avec les besoins de la philosophie des mathématiques, en particulier concernant les dilemmes de Benacerraf.

Pourtant, il y a du travail à faire afin d'obéir aux normes philosophiques de rigueur formelle et de clarifier la position du platonisme pur-sang dans l'ontologie, la théorie de la référence et la théorie de la vérité. 
Jacques CABARET

La maladie animale : théories, expertises et actions

Thèse dirigée par Jean Gayon

Jacques.Cabaret@tours.inra.fr

\section{La maladie dans ses effets, un écart à la norme physiologique ou bien une nouvelle norme pour vivre?}

Les origines de la maladie, ontologique ${ }^{7}$ - un agent extérieur en est la cause ou bien fonctionnelle, une rupture de l'équilibre de l'organisme en est la source -, conditionnent pour beaucoup les aspects thérapeutiques. Pour définir la maladie d'autres approches que les conceptions ontologiques ou fonctionnelles sont nécessaires. Selon Auguste Comte, l'état pathologique ne diffère pas sensiblement de l'état physiologique: l'étude des cas pathologiques devient aussi un moyen de mieux comprendre l'état de santé. Pour Claude Bernard, la maladie est l'expression troublée d'une fonction normale. Les points de vue cependant diffèrent sur les moyens d'acquérir un savoir sur les maladies: Auguste Comte pense que la maladie remplace les expérimentations et Claude Bernard que l'expérimentation physiologique nous renseigne sur la maladie. George Canguilhem ${ }^{8}$ distingue l'anomalie du pathologique : «L'anormal ce n'est pas la pathologie. Mais le pathologique c'est bien l'anormal.» Le contexte de leur apparition les distingue: «L'anomalie éclate dans la multiplicité spatiale, la maladie éclate dans la succession chronologique. » Canguilhem oppose une normativité biologique à une norme de la vie tolérée par le vivant. Il récuse la norme autour des constantes biologiques. Cet énoncé s'oppose à la théorie biostatistique

7. F. Laplantine, Anthropologie de la maladie, Paris, Payot, 1992, p. 55.

8. G. Canguilhem, Le normal et le pathologique, Paris, « Quadrige », PUF, 2011, p. 96-99. Cette édition est une réédition de l'ouvrage de 1943, lui-même légèrement modifié en 1950. 
de Christopher Boorse ${ }^{9}$. En fait Boorse s'appuie sur le physiologique : la maladie « résulte d'un empêchement du fonctionnement normal, [...] d'une réduction des capacités fonctionnelles en dessous de leur efficacité habituelle ». La notion d'individualité de Canguilhem peut être reprise dans un contexte plus large et plus représentatif d'aptitudes selon Nussbaum ${ }^{10}$. Il y a une réelle nécessité de refonder la maladie sur le sujet, le patient animal ou humain, dans son contexte social.

9. C. Boorse, «Health as a theoretical concept», Philosophy of science, 44, (1997), p. $542-573$.

10. M. C. Nussbaum, Frontiers of Justice: Disability, Nationality, Species Membership, Cambridge, MA, The Belknap Press, 2006. 
Sébastien DUTREUIL

L'évaluation philosophique de l'hypothèse Gaïa Thèse dirigée par Jean Gayon et Philippe Huneman seb.dutreuil@gmail.com

\section{Pour quelles raisons cherche-t-on une définition de la vie ? Quel genre de mot est « vie »?}

Après une éclipse partielle entre les années 1960 et 1990, la question de la définition de la vie refait surface dans des disciplines scientifiques: l'astrobiologie, la vie artificielle, l'origine de la vie. La question, pourtant centrale, des motivations qui sous-tendent ces efforts définitionnels a été étrangement délaissée. Pour quelles raisons les astrobiologistes (et d'autres) déploient-ils des efforts importants pour définir la vie?

Nous montrons que deux réponses sont possibles, donnant lieu à deux projets qui n'ont jusqu'alors pas suffisamment été distingués. Le premier considère que la définition de la vie est une fin à laquelle l'astrobiologie doit concourir : la NASA envoie des sondes dans l'espace, finance des expériences de (bio)chimie pour obtenir une définition universelle de la vie que, dit-on, nous ne pourrions obtenir autrement. Le second projet envisage la définition de la vie comme un moyen nécessaire pour répondre à certaines questions scientifiques : chercher l'origine de la vie ou de la vie sur d'autres planètes nécessite, dit-on, une définition de la vie (sans quoi nous ne savons pas ce que nous cherchons).

Il y a dans cette littérature un accord unanime, explicite ou implicite, selon lequel «vie » est un terme de classe : c'est un concept dont les êtres vivants sont des instances, ou bien un terme d'espèce naturelle - natural kind - dont les êtres vivants sont les membres. Deux conceptions de la définition sont dès lors disponibles : (i) une liste des propriétés nécessaires et suffisantes d'appartenance à la classe, (ii) la spécification des propriétés 
essentielles à l'espèce naturelle suivant une conception héritée de Kripke et Putnam.

La tâche se donnant la définition de la vie comme fin doit faire face à deux types d'objection : peut-on définir la vie ? Doit-on le faire ? Le premier type d'objection a essentiellement reposé sur le caractère extensionnellement vague de la vie ou sur le fait que les êtres vivants évoluent. La seconde objection repose tantôt sur une conception normative des sciences (la science doit-elle avoir pour ambition ultime de fournir des définitions ?), tantôt sur l'idée que le projet d'une définition de la vie est voué à l'échec compte tenu de la diversité des contextes théoriques dans lesquels il est entrepris.

Le recours à la définition de la vie comme moyen fait face à des objections distinctes. On doit en effet ici d'abord s'interroger sur la question de savoir si c'est bien d'une définition que les scientifiques ont besoin pour leur enquête. Nous montrons que non en examinant l'ensemble des contextes dans lesquels on a prétendu le contraire : une recherche de biosignatures (ou de l'origine de la vie, ou de vie ailleurs) ne requiert que des critères suffisants d'appartenance à la classe, une réflexion sur l'habitabilité des planètes seulement des critères nécessaires.

Nous proposons enfin de réviser l'idée acceptée jusque-là que « vie » est un terme de classe en proposant au contraire qu'il sert à désigner un individu (l'ensemble des «êtres vivants» liés entre eux par des relations généalogiques). Cette thèse à propos de la vie est analogue (mais logiquement indépendante) à la thèse que Ghiselin et Hull soutenaient à propos des espèces biologiques (species). Selon notre analyse, qui rend compte des rares usages théoriques qui sont faits de "Vie», Vie serait soit un nom propre (il n'a donc pas de signification) soit un concept singulier mono-instancié.

Deux conclusions donc: (i) il est important de distinguer plus explicitement qu'on a coutume de le faire les projets dans lesquels s'insère une définition de la vie (fin ou moyen ?) compte tenu de la différence des problèmes philosophiques auxquels ils doivent faire face, (ii) nous proposons de réviser l'idée que «vie » soit un terme de classe et montrons que son usage s'accorde mieux avec l'idée qu'il sert à désigner un individu. 
Edgar Fernando GÁLVEZ

Thèse dirigée par Marco Panza

ferchogalvez@yahoo.com

\section{Sur l'objet mathématique et la réalité mathématique dans le cadre du structuralisme non éliminanif : le débat Shapiro-Parsons}

Le structuralisme mathématique est une perspective philosophique qui a émergé au $\mathrm{XX}^{\mathrm{e}}$ siècle au sein du débat concernant le problème des «réductions multiples", apparu au cours des $\mathrm{XIX}^{\mathrm{e}}$ et $\mathrm{XX}^{\mathrm{e}}$ siècles (Dedekind, Cantor, von Neumann et Zermelo, entre autres). On sait, par exemple, que dans le cadre des deux théories des ensembles différentes on peut affirmer les identités «» et «». Pourtant, on n'a que «». À cet égard, Benacerraf (1965) conclut que: si les mathématiques sont réductibles au langage des ensembles, et s'il n'y a pas un unique ensemble correspondant aux nombres naturels, les nombres naturels ne sont alors pas du tout des objets. Ils ne sont qu'une structure de progression, dont les relations sont les seules conditions qui comptent.

Face à cette perspective, dite éliminative, il y a des réponses structuralistes non éliminatives : par exemple, celles de Stewart Shapiro (Structuralisme ante rem - SAR) et de Charles Parsons (Structuralisme post rem - PRS). Le but est alors de montrer que parmi ces deux perspectives existent pourtant des différences profondes.

D'après Shapiro, il est bien possible d'offrir une explication structuraliste des mathématiques, gardant la référence aux objets (des objets de «bonne foi »). Son ontologie s'appuie sur deux postulats : «la mathématique est la science des structures » et "les objets mathématiques sont des places dans une structure ». Ainsi, les structures sont les objets 
prioritaires des mathématiques et sont définies comme la forme abstraite de tous les systèmes qui les exemplifient. À ce titre, on formule l'existence d'un univers de structures, ayant de bonnes conditions d'identité. De même, les objets de la structure, étant ses places, ont des conditions internes d'identité, dont Shapiro explique à travers la différence entre les perspectives «les places-sont-objets » et « les places-sont-rôles ». Selon la première, l'identité «" porte sur une identité entre places d'une même structure, ce qui garantit la référence à de vrais objets mathématiques. Par contre, des identités telles que «» ou «» n'ont pas de sens dans la structure des nombres naturels, ce sont plutôt des identités du type « les places-sont-rôles ».

En revanche, le structuralisme de Parsons s'érige comme une conception structuraliste des objets mathématiques et non pas comme une conception structuraliste des mathématiques. Pour Parsons la notion de structure du SAR ne peut rendre compte que d'objets abstraits purs, et non pas d'autres objets abstraits comme les objets quasi-concrets. Les structures ne sont pas considérées comme des objets ontologiquement prioritaires. À la différence des objets purs, les objets quasi-concrets sont des objets d'intuition, car ils portent une représentation intrinsèque. Le langage des tokens et des types et le langage de traits de Hilbert sont deux exemples d'objets quasi-concrets. L'enjeu ici est qu'un objet abstrait pur peut devenir « intuitionnable » par le biais d'un objet quasi-concret. Les nombres naturels sont un cas paradigmatique. À cet effet, Parsons prend une notion de structure, différente de celle de Shapiro, qu'il appelle métalinguistique. Dans ce cadre, la structure des nombres naturels peut être décrite d'une façon plus concrète par le biais d'un modèle intuitif, tel que les chaînes de traits de Hilbert, par exemple. De ce point de vue, à la différence du SAR, l'unicité de la structure n'est pas essentielle à l'explication structuraliste d'une classe d'objets. Bien au contraire, il existe plusieurs modalités de référence aux nombres naturels.

\section{Bibliographie}

Benacerraf, P., «What the numbers could not be?», The Philosophical Review, 74 (1), (1965), p. 47-73.

Dedekind, R., La création des nombres, introduction, traduction et notes par Hourya Benis Sinaceur, Paris, Vrin, 2008.

Parsons, C., «Structuralism and metaphysics », The Philosophical Quarterly, (54) 214, (2004), p. 56-77.

Parsons, C., Mathematical Thought and Its objects, Cambridge, Cambridge University Press, 2008.

Shapiro, S., Philosophy of Mathematics : Structure and Ontology, Oxford, Oxford University Press, 1997.

Resnik, M., Mathematics as a Science of Patterns, Oxford, Oxford University Press, 1997. 
Eylem HACIMURATOĞLU

La fondation husserlienne des sciences de la nature

Thèse dirigée par Renaud Barbaras

e.hacimuratoglu@gmail.com

\section{L'idéalité comme condition de possibilité de la connaissance en général et d'une métaphysique légitime}

Les Recherches Logiques de Husserl, l'œuvre de percée de la phénoménologie, se manifestent comme une critique de la connaissance qui reconnaît dans l'idéalité la condition de possibilité de la connaissance en général. Husserl prend pour l'idéal le contenu qui puisse être répété identiquement dans les différents actes de conscience, telle que la signification d'une expression qui ne peut pas être réduite à l'acte même de l'expression. Cependant, cette irréductibilité de la signification aux actes d'expression, ne revient pas à dire nécessairement qu'elle a une existence totalement en dehors de la conscience. De même que Husserl se refuse à réduire la signification à un contenu psychique ajouté de manière associative au mot et qui le doterait de sens, de même il s'abstient de l'hypostasier comme quelque chose qui viendrait d'un autre règne extra-mental pour adhérer au mot (le mythe de la signification). En acceptant le caractère idéal et identique de la signification, il la considère dans sa relation avec l'acte de signifier et analyse les expressions comme des actes dans lesquelles les significations se manifestent comme unités idéales.

La théorie de la signification dans les Recherches Logiques qui vise à trouver une voie moyenne entre hypostase psychologisante et hypostase métaphysique, a de certaines implications pour une conception juste de l'intentionnalité. D'une part, l'acte de signifier qui confère à l'expression sa signification ainsi que son rapport à l'objet indépendamment du fait que 
son objet existe véritablement ou non, joue le rôle de prototype de toute intentionnalité. D'autre part, le fait que le premier Husserl considère la relation entre l'acte réel de signifier et la signification idéale comme celle entre un individuel instanciant et un universel instancié, tout comme le rouge en espèce et le moment rouge d'un objet, permet de concevoir le rapport à l'objet comme un moment dépendant ou une propriété intrinsèque de l'acte intentionnel. Cette propriété de l'acte confirme que celui-ci se dirige déjà et immédiatement vers un objet sous diverses modalités. Cette orientation de l'acte vers son dehors permet de déterminer une ouverture qui dépasse l'immanence réelle de l'acte vers une immanence intentionnelle comme une transcendance. La signification en tant qu'unité intentionnelle de l'acte habitera cette transcendance dans l'immanence. En somme, le fait que la signification se pose comme une entite heimatlos par rapport au mythe de la signification donne naissance à l'idée d'une relation immédiate entre conscience et monde.

Ainsi s'ouvrirait pour nous la possibilité d'une métaphysique légitime dans le cadre de la phénoménologie husserlienne. L'intentionnalité en tant que rapport immédiat de la conscience à son objet, grâce à ces diverses modalités, produit de nouveaux types d'objets, dont les significations idéales. Les objets idéaux, comme les objets réaux, sont donnés dans certains modes des actes de conscience. Ils sont ce qu'ils sont dans ces actes qui les visent. Là où la métaphysique traditionnelle considère l'être comme un absolu et un prédicat réal des objets, la phénoménologie nous offre une conception critique de l'être selon laquelle l'être des objets n'est qu'être visé au sens où l'être n'est pas un prédicat réal mais une modalité qui renvoie au mode de donation des objets. Ainsi, la signification pourrait être traitée comme un être idéal qui se donne elle-même dans l'abstraction idéalisante. Nous croyons que le mérite de cette lecture qui souligne la question de l'idéalité à partir de la signification, ce serait de nous mettre dans la voie d'une métaphysique légitime. 
Max HARDT

Des arts libéraux à l'encyclopédie, l'invention philosophique d'une culture moderne (XVI ${ }^{e}-X V I I I^{e}$ siècle)

Thèse dirigée par Denis Kambouchner max.hardt@,hotmail.fr

\section{Les méthodes de Claude Lancelot, entre pédagogie et philosophie du langage à Port Royal}

Il s'agit d'aborder ici les enjeux proprement philosophiques des textes publiés par Claude Lancelot, membre de la communauté des «Solitaires » de Port-Royal, coauteur avec Antoine Arnauld de la Grammaire générale et raisonnée (dite «Grammaire de Port-Royal»), et éminent pédagogue des Petites Écoles, qui rédigea durant ses années d'exercices plusieurs manuels, appelés " Méthodes ", destinés à faciliter l'apprentissage du latin, du grec, de l'espagnol et de l'italien. Il collabora également avec Pierre Nicole à l'édition d'un manuel de vocabulaire grec dont la forme versifiée devait faciliter la mémorisation (le Jardin des Racines grecques), ainsi qu'à des traités, manuels et anthologies consacrés à la poésie (l'Epigrammatum delectus en particulier).

Outre que ces manuels et traductions constituent en eux-mêmes de précieuses sources pour l'étude des pratiques pédagogiques expérimentées en marge des institutions jésuites dominantes à cette époque, les préfaces et « avis au lecteur » rédigés par Claude Lancelot comportent des remarques générales particulièrement instructives quant à l'approche de l'enseignement des langues et des textes en langue étrangère à Port-Royal. Or cette thématique ne peut être indifférente à un contexte intellectuel et spéculatif dans lequel les Messieurs accordaient à la théorie du langage et à la logique une place centrale, que ce soit pour l'élaboration d'une philosophie de la connaissance, ou dans le cadre de réflexions sur la morale, le salut, et 
l'interprétation des Écritures dont dépend le sens de ces derniers. Je voudrais ainsi proposer un aperçu de l'éclairage singulier que les textes pédagogiques et théoriques de Lancelot apportent sur certaines des thèses défendues à Port-Royal sur les interactions subtiles de l'intellect, du langage et de la volonté, au travers d'un discours mêlant les héritages d'Augustin, de Descartes et de Pascal.

Le concept central pour comprendre l'originalité de la pensée de Claude Lancelot est celui «d'usage » appliqué aux langues : en effet, les manuels de Port-Royal insistent sur le fait que l'usage apparaît comme un «sage maître », dont les écarts par rapport aux règles brutes de la grammaire ne doivent pas être compris comme des irrégularités arbitraires, mais comme le signe d'un esprit alerte et, à cet égard, exemplaire. La pratique des auteurs anciens induite par cette perspective singulière confirme le fait que la vision portée par les Messieurs de Port-Royal sur les textes antiques dépasse l'idée d'une condamnation morale et religieuse sans appel de la sagesse païenne : on y discerne la reconnaissance d'une authentique vertu pédagogique, qui s'appuie sur l'étude des singularités de chaque auteur, indépendamment de la valeur religieuse de ses assertions. C'est là une approche novatrice et minutieuse de la valeur éducative du style et de la fréquentation du « naturel » littéraire, que Port-Royal place au service de sa propre pédagogie de l'exemplarité. 
Anne-Sophie JOUANNEAU

Le désir chez l'Avicenne latin

Thèse dirigée par Jean-Baptiste Brenet

annesophie.jouanneau@sfr.fr

\section{Un traité de médecine est-il une illustration métaphysique ? L'exemple de l'amour chez Avicenne}

Les Arabes ont non seulement légué aux Latins des traités de philosophie mais aussi des ouvrages de médecine : le Canon de médecine d'Avicenne fut ainsi traduit au XII ${ }^{\mathrm{e}}$ siècle à Tolède à la même époque que sa somme philosophique (le Kitāb al-Šifä', qui sera la Sufficientia des Latins, incluant notamment une Métaphysique et un Traité sur l'âme). Ces deux corpus que les Latins ont commentés, parfois dans les mêmes ouvrages, entretiennent un rapport qui ne cesse d'être interrogé : cette médecine est-elle subordonnée aux principes métaphysiques ou bien au contraire l'expérience, sur laquelle la pratique médicale repose, a-t-elle infléchi certaines thèses métaphysiques ? Ce rapport pose ici question à partir du thème de l'amour présent, de manière surprenante, à la fois dans la médecine et la philosophie. Porté par le même terme arabe de 'išq, ce thème semble revêtir deux sens contraires : dans la métaphysique, l'amour est le principe du mouvement des sphères célestes; tandis que dans le Canon, il désigne une pathologie.

Avicenne ne serait-il que le simple héritier de deux topoï qu'il utiliserait sans se soucier de leur cohérence d'un corpus à l'autre? En effet, d'une part les commentateurs arabes d'Aristote avaient déjà utilisé ce terme de ' $i \check{s} q$ pour rendre compte du mouvement des cieux; d'autre part, le ǐ̌q que Gérard de Crémone, traducteur du Canon, transcrit en latin par ilisci, s'inscrit dans la tradition antique, reprise par les médecins arabes, de l'amour 
mélancolique. L'Occident latin connaîtra plus généralement cette tradition sous la dénomination d' "amour héroïque » - dénomination due au latin amor hereos que l'on trouve dans une traduction de Constantin l'Africain se référant au même cas, et qui donnera lieu à de nombreux commentaires croisant les différentes sources arabes dont le Canon.

Topos positif d'un côté, négatif de l'autre : si donc ces deux « amours » semblent être deux théories inconciliables, serait-ce là le signe d'une discontinuité du système avicennien, scindé entre médecine et philosophie ? Bien au contraire, une lecture précise des passages tirés de la Métaphysique et du Canon de médecine permet de mettre en évidence la continuité, élaborée terme à terme par Avicenne, entre les deux doctrines. C'est notamment le rôle de l'imagination qui fait lien entre les âmes humaines et les âmes célestes, elles aussi dotées d'imagination. Il se dégage de cette analogie une conception de l'amour où la pathologie, en tant que pathologie, est commensurable à l'amour supralunaire, en tant que perfection. Le principe métaphysique ne se trouve pas infléchi par l'observation médicale, ni a fortiori contredit par lui : la lecture croisée autorise bien plutôt à voir dans le cas médical de l'amour une illustration métaphysique de ce principe placé au sommet de l'édifice avicennien. 
Pierre de JOUVANCOURT

Enjeux philosophiques de l'Anthropocène: nature, histoire et modernité

Thèse dirigée par Bernadette Bensaude-Vincent pierre.dejouvancourt@gmail.com

\section{Anthropocène : histoire, nature et modernité}

L'Anthropocène est un concept selon lequel les temps que nous vivons seraient caractérisés, du point de vue géologique et stratigraphique, par la prépondérance de l'activité humaine sur le reste des processus biogéochimiques. L'Anthropocène est ainsi un candidat à la succession de l'Holocène dans la géochronologie et la stratigraphie, ce qui n'est pas sans provoquer d'importants débat au sein des sciences de la nature et des sciences humaines traitant de questions environnementales. En premier lieu, le concept d'Anthropocène possède un caractère massif, puisqu'il vise à la fois de l'entièreté du globe (la «nature »), de l'histoire et du devenir de l'espèce humaine, ce qui ne manque pas de soulever des difficultés narratives. C'est aussi une saisie, en un seul tenant conceptuel, de toutes les alertes environnementales qui concernent de près où de loin les affaires humaines et donc, virtuellement, de tous les problèmes qui en ont été tirés. C'est également, du point de vue épistémologique, un concept qui force un questionnement sur les pratiques et les frontières des expertises historienne et scientifique.

Du point de vue historique, l'Anthropocène fait entrer une tension entre téléologie, linéarité et histoire "désorientée ». Bien que cette question ne soit pas nouvelle, elle rouvre un débat qui pourrait avoir des conséquences importantes sur les pratiques de la recherche (surtout dans les pays de langue anglaise). Elle se structure autour de deux questions : "comment faire de l'histoire avec des «faits » venus des sciences sans perdre de vue l'action 
humaine ? » puis : « comment écrire l'histoire du point de vue de ce devenir géologique qui s'impose? »

L'Anthropocène exige également une réactualisation ou un renforcement des questions autour du concept de nature, questions qui ont notamment travaillé l'écologie politique, l'anthropologie et de l'éthique environnementale. En effet, la disparition manifeste des régions du monde qui pouvait être qualifiées de "naturelles» ou «non artificielles» et l'émergence des produits récents de la technique (nanotechnologies et biologie de synthèse notamment) ont de façon irréversible dévalué la double frontière nature/artifice et nature/humain.

Enfin, l'Anthropocène change, rétrospectivement, le regard qui peut être porté sur la modernité. Ce qui semble réunir bien des problèmes posés par la pensée environnementale, est cette interrogation quasi-permanente sur l'agir technique de masse de la modernité. Parfois en tant que point de départ, d'autres fois en tant que point d'arrivée, celui-ci constitue de façon invariable un point de passage. 
Daniel LAROSE

La méthodologie des cosmologies de Platon et d'Aristote Thèse dirigée par Luc Brisson et Louis-André Dorion quebec104@hotmail.com

\section{La preuve de la sphéricité de l'univers (Timée 33b 1-7; De caelo II 4)}

Les cosmologies de Platon et d'Aristote, telles qu'exposées dans le Timée et le De caelo, partagent une caractéristique commune : la conviction que l'univers - que les Grecs appelaient «kosmos » en vertu de son ordre et, par conséquent, de sa beauté - est un être "divin ». L'univers est « divin » dans la mesure où on lui attribue deux caractéristiques essentielles de la divinité : l'immortalité et la perfection. Dans le Timée (33b 1-7) et le De caelo (II 4), Platon et Aristote, en s'appuyant sur la perfection présumée de l'univers, lui attribuent une forme sphérique. Leur preuve se laisse résumer ainsi : P1) l'univers est parfait, P2) la sphère est la figure parfaite, C) l'univers est sphérique. La preuve de Platon repose sur un mythe vraisemblable (eikôs muthos). D'après ce mythe, un démiurge divin a donné à l'univers la figure qui lui était apparentée : la sphère. À l'univers, vivant qui enveloppe tous les vivants, dieu parfait (teleon, achevé et complet), convenait la figure où s'inscrivent toutes les autres figures, la sphère, figure la plus parfaite et la plus semblable à elle-même puisque son centre est équidistant de tous les points de la périphérie. Tout en refusant de recourir au mythe, Aristote n'en reprend pas moins, faute de moyens d'observation, l'essentiel de la preuve de Platon : l'univers est sphérique parce que c'est la forme appropriée à son essence et la première par nature. Au corps parfait (teleon), achevé et complet, convient une figure parfaite. Certes, le Stagirite s'appuie également sur trois autres arguments fondés sur des considérations 
géométriques, physiques et téléologiques, mais certains indices montrent qu'il accorde plus d'importance à l'argument à partir de la perfection de l'univers. Premièrement, cette primauté est illustrée par le ton du chapitre. Dans l'introduction et la conclusion, Aristote insiste sur la perfection de l'univers en des termes qui renvoient à la preuve de Platon. Deuxièmement, la taille de cet argument montre son importance : 36 lignes dans le texte grec, ce qui équivaut presque à la taille des trois autres arguments (39 lignes : $12+8+19$ ). Troisièmement, Aristote qualifie sa thèse de la sphéricité de l'univers de "pistis»(287 a 30-31). Dans ce contexte, ce terme, que l'on peut traduire par "conviction", doit être compris comme une conviction non scientifique puisqu'il se fonde sur un principe vraisemblable: la perfection de l'univers. C'est, en effet, le constat d'Aristote lorsqu'il récapitule les résultats du livre I du De caelo au début du livre II (283 b 26-284 a 2). Cette opinion est également partagée par Simplicius dans son commentaire au De caelo $(369,14)$. Ainsi, Aristote, faute de moyens d'observation, ne peut élever sa preuve de la sphéricité de l'univers au-dessus du mythe vraisemblable du Timée de Platon. 
Manuel LEVAL-DuCHÉ

L'« attitude réaliste » : enquête sur les équivocités du réalisme en philosophie contemporaine, à partir de Merleau-Ponty

Thèse dirigée par Jocelyn Benoist manuel.levalduche@gmail.com

\section{Merleau-Ponty et les deux voies d'un réalisme anthropologisé}

Nous voudrions traiter l'œuvre de Merleau-Ponty comme exemple et théâtre de l'équivocité, voire de la concurrence, entre deux usages de l'étiquette «réaliste» en philosophie, dont la fortune dépasse de loin les seuls textes merleau-pontiens, et qui ont en commun de neutraliser le problème du «réalisme» considéré comme option spéculative ou métaphysique parmi d'autres dans le cadre d'une investigation sur l' « essence de la réalité », et de proposer à ce titre deux stratégies possibles pour un déplacement « anthropologique » dudit problème.

Le premier « lieu » réaliste que nous voudrions travailler est celui d'une stratégie philosophique consistant à exempter ou à soustraire une certaine catégorie d'expérience (ou d'énoncés) du jeu ordinaire du doute et de la dispute, et au fond de la vérité et de la fausseté. Nous serons amenés pour ce faire à exploiter la fécondité philosophique, pour décrire un tel niveau de certitude, d'une véritable sémantique (voire rhétorique) de l'immédiateté, du préfixe (« pré- », « Ur- », « archi- », etc.), du « toujours déjà », du « fond » (« toile de fond », « arrière-plan », « sol ») et du « hors-thème » (du tacite, de l'implicite, du « cela va sans dire »), qui constitue le fonds commun d'une certaine analyse du «plain-pied» silencieux du réel ou de l'«avoir-déjà » des choses.

Mais il nous semble possible, à partir de l'œuvre de Merleau-Ponty, de suivre une ligne réaliste alternative : en effet, le réalisme au sens du 
naturalisme de la pensée objective, de l' « en-soi-pour-nous » corrélatif de la spontanéité véritative de la perception (Wahrnehmung), est l'objet, chez le phénoménologue français, d'une destitution théorique, mais aussi et surtout d'une « réhabilitation» existentielle ou anthropologique, puisque MerleauPonty produit la genèse de cette spontanéité comme attitude ou performance humaine fondamentale, fonction de décentrement, de négation de soi, d'objectivation, d'universalisation et d'hypostase ( «le vrai et unique monde ») spontanées, selon la perspective d'un réalisme non plus doctrinal mais vital ou existentiel - celui, précisément, de l'attitude naturelle.

La question se posera donc de savoir comment cette « attitude réaliste » spontanée du vivant humain s'articule avec la certitude primordiale du monde (si, comme nous en faisons l'hypothèse, ce sont là les deux pans, parfois difficiles à démêler, de la «foi» perceptive) : si la seconde fait l'objet d'une description qui n'autorise pas le regard d'un «spectateur étranger », désengagé de cette certitude posée comme irréductible, il est possible au contraire d'engendrer la première (l'attitude réaliste, d'un réalisme qu'on pourrait dire incorporé, anthropologisé voire naturalisé), comme fonction, depuis le lieu de son absence, à partir d'un état où elle ne serait pas donnée. 
Charles NKOURISSA

Langage et souveraineté chez Thomas Hobbes

Thèse dirigée par Chantal Jaquet charleslebonnkourissa@yahoo.fr

\section{Le langage dans la méthode résolutive compositive : de Galilée à Hobbes}

Il est question d'examiner les éléments qui justifieraient un rapprochement entre Galilée et Hobbes, considérés comme deux initiateurs de la révolution scientifique, l'un en physique et l'autre en science politique. L'analyse sera menée à partir de leur démarche méthodologique essentiellement définie par les mathématiques qui sont pour les deux auteurs la norme de la démonstration parce que révélatrices de vérité dans leur contenu et dans leur forme. En cela, l'accent sera mis sur l'expérience comme élément déterminant de la certitude du raisonnement chez les deux auteurs dans la mesure où elle permet de partir des principes et des causes de la connaissance humaine pour en déduire la connaissance des choses singulières tant en physique qu'en science politique. Cette expérience fera l'objet de la rupture des deux auteurs avec la tradition aristotélicienne qui s'appuie sur la logique formelle comme certitude du raisonnement tandis que seules les mathématiques en rapport à la physique, constituent la méthode de pensée la plus rigoureuse, capable de donner toute sa valeur à la connaissance de la nature physique pour Galilée et Hobbes. Ensuite, nous établirons les éléments divergents dans la démarche des deux auteurs. Pour Galilée l'objet de la méthode est la nature physique, en revanche pour Hobbes les passions et les intérêts des hommes. Dès lors nous passons de la mathématisation de la nature à la probabilité de la mathématisation du langage politique. Toutefois, serait-il légitime d'évoquer la scientificité 
de cette méthode chez Hobbes où elle se déploie dans les passions qui sont imprévisibles? Serait-il question de la même méthode résolutive compositive de Galilée à Hobbes ? 
Delphine OLIVIER

Prévention et prédiction : comment penser l'anticipation dans les sciences médicales? Enquête historique et épistémologique

Thèse dirigée par Jean Gayon delphine_olivier@hotmail.com

\section{De la prévention à la prédiction : la médecine est-elle parvenue à une nouvelle connaissance de l'individu?}

À la fin du $\mathrm{XX}^{\mathrm{e}}$ siècle, la prévention des maladies tente de se développer dans un cadre nouveau: celui d'une médecine devenue "prédictive ». Il s'agirait de recourir à des informations biologiques individuelles afin de prédire à chaque individu son risque personnel de développer une maladie donnée. Ce faisant, les promoteurs de cette médecine ont explicitement cherché à rompre avec une médecine préventive dont la cible principale serait des populations plutôt que des individus. Le but de cette présentation est de montrer que la pensée de l'individu a été cruciale dans l'élaboration des projets de médecine prédictive.

Nous commençons par montrer que le développement des premiers projets de médecine prédictive (tournant des années 1960-1970) est concomitant d'un intérêt renouvelé de la médecine pour l'individu. Explication de la maladie orientée vers la notion de « terrain » plutôt que sur des éléments extérieurs (germes de la maladie), mise en avant de la question de l'inégalité des hommes devant la maladie, importance des découvertes biologiques qui ont contribué à forger la notion d' « individu biologique », volonté de mettre en œuvre une médecine préventive personnalisée : tels sont les éléments qui placent l'individu au cœur des préoccupations de la médecine. 
Nous montrons ensuite que cette pensée de l'individu conduit à deux manières distinctes d'envisager la prédiction. D'une part, le constat d'une double hétérogénéité - à savoir l'inégalité des hommes devant la maladie et la variation interpersonnelle des constituants biologiques - conduit à rechercher des corrélations entre des biomarqueurs et la survenue des maladies. D'autre part, on voit à la même époque surgir une conception plus dynamique de la prédiction. Les progrès de la médecine de laboratoire permettant de se tourner vers l'intérieur de l'organisme, on voit surgir l'idée qu'une détection précoce de tendances dégénératives est possible. La prédiction prend alors le sens d'une anticipation des symptômes par un examen attentif des données biologiques.

Nous terminons cette présentation par un bref examen des problèmes qui se posent à cette médecine prédictive, du fait même de son orientation vers l'individu. La première question est celle de l'accès à une connaissance directe des données biologiques individuelles. Si le progrès des analyses de laboratoires et le développement de nouveaux modèles statistiques sont envisagés comme moyen de surmonter cet obstacle, la médecine prédictive doit encore affronter deux autres questions. D'une part, l'interprétation des données biologiques individuelles en termes de santé pose la question de l'existence et de la définition d'une norme individuelle de santé. D'autre part, la médecine prédictive prend le risque d'une possible réduction de l'individu à ses composantes infra-organiques, au détriment d'une approche plus globale de l'individu. 
Kotaro ONO

La morale et la société - Bergson et la science des mours

Thèse dirigée par Michel Bourdeau onokotaro01@gmail.com

\section{Deux morales sociales - Bergson et Durkheim}

La morale est-elle sociale? Deux intellectuels français du début du $\mathrm{XX}^{\mathrm{e}}$ siècle, Henri Bergson (1859-1941) et Émile Durkheim (1858-1917), ont partagé le même intérêt pour cette question. Tous deux affirment que la morale est inséparable de la société. Bergson précise sa position dans Les deux sources de la morale et de la religion (1932), alors que Durkheim ne cesse de s'intéresser à la question morale depuis ses premières études jusqu'à la fin de sa vie. Pour comprendre les points de convergence et de divergence entre ces deux auteurs, deux questions se doivent d'être traitées. Quel est l'impact de la distinction entre morale close et morale ouverte, telle qu'elle a été introduite par Bergson quant au rapport entre la morale et la société ? Et, deuxième question, comment justifie-t-il cette distinction?

En ce qui concerne la première question, deux réponses sont possibles. Premièrement, selon Bergson, il y a deux sortes de relations entre la morale et la société, selon qu'elles se réfèrent à la morale close ou à la morale ouverte. D'une part, dans le cadre de la morale close, la position de Bergson se rapproche de celle de Durkheim, parce que, pour eux, moraliser l'individu est le socialiser intérieurement pour qu'il s'attache à la société et qu'il soit par conséquent solidaire aux autres. D'autre part, dans le cadre de la morale ouverte, Bergson s'éloigne de Durkheim, parce que, selon ce dernier, un individu, s'il est un réformateur moral, s'attache intérieurement à une nouvelle société, en train d'apparaître, pour transformer la société présente, alors que, selon Bergson, il ne s'attache plus intérieurement à la société, 
mais il s'attache à la "vie en général» pour la transformer. Ce qui est important, c'est que, selon Bergson, grâce à cet attachement à la vie en général, le réformateur moral communique avec des personnes par l'amour divin comme une émotion supra-intellectuelle, bien qu'il ne s'attache plus intérieurement à la société. Deuxièmement, la distinction entre la morale close et la morale ouverte est plus fondamentale que la distinction traditionnelle entre l'égoïsme et l'altruisme adoptée par Durkheim. Remarquons qu'il y a deux sens au mot «clos». Dans un premier sens, clos signifie la fermeture du groupe. La morale close nous fait toujours prendre « une attitude qui est celle de la discipline devant l'ennemi ». Dans un second sens, clos signifie la coïncidence naturelle et circulaire entre l'intérêt particulier et l'intérêt général. Ce qui est important, c'est que le cercle entre l'individu et la société ne peut pas s'étendre jusqu'à l'humanité entière, parce qu'il y a une distinction fondamentale entre la patrie et l'humanité en morale. La distinction entre l'égoïsme et l'altruisme ne marque pas cette distinction, en faisant supposer qu'une même tendance altruiste grandit depuis la famille jusqu'à l'humanité par la patrie.

En ce qui concerne la deuxième question, la distinction entre la morale close et la morale ouverte de Bergson repose sur l'observation de la guerre. En temps de guerre, "les devoirs envers l'homme en tant qu'homme », c'est-à-dire la morale ouverte, se révèle comme le contraire de l'exigence fondamentale de la vie sociale, parce que le meurtre et le pillage deviennent facilement licites et méritoires dès que la guerre commence. La réflexion de Bergson part de ce fait. En effet, d'une part, Durkheim ne voit pas vraiment l'antagonisme entre les trois sentiments collectifs conformes aux trois groupes sociaux (la famille, la patrie et l'humanité), d'autre part, il remarque lui aussi qu'il y a un conflit entre le «patriotisme » et le « cosmopolitisme ». Alors que Durkheim oscille entre la continuité entre le sentiment national et le sentiment humain et la discontinuité entre ces deux sentiments, il est évident, pour Bergson, que l'amour de la patrie doit être distingué de l'amour de l'humanité. La philosophie morale de Bergson consiste à trouver une différence de nature entre eux et à révéler une autre origine à la morale que la société, en allant jusqu'au bout de l'antinomie entre ces deux sentiments. 
Elena PARTENE

Finitude et finalité chez Kant Thèse dirigée par Christian Bonnet elena.partene@gmail.com

\section{Image et signe dans la troisième Critique}

Le projet critique vise l'élucidation des conditions et des limites de l'objectivité. Or, la question de la possibilité de l'objectivité naît, chez Kant, de l'hétérogénéité originaire de l'intuition et du concept, du sensible et de l'intelligible. Il s'agira donc de mettre au jour les actes qui effectuent l'objectivation: la synthèse et la réflexion seront les deux opérations permettant de lier sensible et intelligible. Cependant elles présupposent par là même que le divers se prête à l'objectivation, que le sensible, pour être alogique, n'est pas antilogique, que le donné matériel ne repousse pas l'acte qui l'informe. Y a-t-il une expérience qui puisse l'attester? Y a-t-il une expérience qui, suspendant l'objet, présente un accord du réel et du sujet? Que signifie pour le sensible de se prêter à l'intelligible, de se possibiliser comme intelligible? Il s'agit de penser un accord pré-objectif de l'intuition et du concept. Au lieu de faire descendre le concept dans le sensible, c'est le sensible qui s'élèverait; au lieu de subsumer le divers, c'est le divers qui se « sursumerait». Cette expérience d'une désensibilisation du sensible semble être celle qui se formule dans le jugement esthétique. En effet, dans la première partie de la Critique de la faculté de juger, on ne se demande plus comment la représentation constitue l'objet, mais comment le donné se prête à la représentation. Ce nouveau questionnement, plus radical puisqu'il concerne le mode de donation du sensible au sujet, inverse la problématique du premier : d'une part, il s'agira de montrer non plus comment les facultés investissent le donné en vue de l'objet, mais comment le donné lui-même se 
présente relativement aux facultés du sujet. Le donné est réfléchi dans son rapport au sujet, d'où une première conséquence : la suspension de l'objet lui-même dans son être pour libérer son apparaître. D'autre part, dès lors qu'il s'agit bien d'une appréhension subjectivante et non objectivante, le jugement sera lié à la subjectivité du sentiment de plaisir et de peine : l'aptitude ou l'inaptitude du sensible à la représentation affecte le sujet selon ces deux modalités pathétiques. Or c'est bien cette expérience affective de la représentabilité du donné qui fonde les deux formes du jugement esthétique : celle du beau et celle du sublime. Dans le beau, le donné est réfléchi relativement à l'imagination et à l'entendement, et le plaisir provient d'un réel qui fait image. L'image est en effet une déréalisation du sensible : l'objet beau est désincarné et devient objet de contemplation. Dans le sublime, le donné est réfléchi relativement à l'imagination et à la raison : le déplaisir initial d'une contrariété (l'impossibilité de configurer l'objet) suscite un plaisir supérieur, qui provient d'un réel qui fait signe. Le signe est en effet la négation de l'image : le spectacle est sublime quand il refuse d'être synthétisé en une forme et se transforme en signe du suprasensible. Ainsi, dans le beau, la représentabilité est vécue comme image, dans le sublime, comme une image qui se nie comme telle, c'est-à-dire comme signe. Dans les deux cas, le sujet fait bien l'expérience d'un sensible qui de lui-même se désensibilise, donc d'un pressentiment de l'Idée à même le sensible. 
Juliette Roussin

Autorité et évaluation des choix démocratiques à l'aune des conceptions procédurales et substantielles de la démocratie

Thèse dirigée par Jean-Fabien Spitz juliette.roussin@gmail.com

\section{Un constitutionnalisme démocratique est-il possible?}

La démocratie tire pour nous sa valeur de ce qu'elle est le gouvernement $\mathrm{du}$ peuple, garantissant à chaque citoyen un statut politique égal et un contrôle général sur les décisions politiques collectives. Mais la démocratie est plus à nos yeux qu'une simple règle de décision égalitaire : son attrait lui vient aussi essentiellement des principes et normes morales et politiques qu'elle incarne et qui, à leur tour, la définissent et lui donnent sa direction. C'est à la démocratie constitutionnelle que nous accordons de la valeur, en d'autres termes: un régime dont le cadre politique et moral est fixé par une constitution, qui stipule de quels droits les individus peuvent se prévaloir, et quels principes politiques doivent être protégés. Comme le suggère Ronald Dworkin, la constitution ne fonctionne pas ici comme une contrainte externe qui pèserait sur la volonté populaire ; elle doit bien mieux être comprise comme l'un des éléments nécessaires pour que l'on puisse véritablement parler d'un régime démocratique.

Afin que cette description de la démocratie constitutionnelle soit correcte, cependant, il faut que les citoyens ou leurs représentants disposent d'un certain pouvoir de définir et de redéfinir ces normes et principes constitutifs de leur démocratie. Or, la plupart des démocraties recourent au contraire à des cours constitutionnelles pour contrôler la conformité des lois et décrets émis par les pouvoirs législatif et exécutif à la constitution. En considérant que le contrôle juridictionnel de constitutionnalité est le meilleur moyen de protéger les principes constitutionnels dans une démocratie, le 
constitutionnalisme contemporain accorde à «l'autorité interprétative » d'un petit nombre de juges la prééminence sur les citoyens et leurs représentants. Ceci semble entrer en contradiction directe avec l'idée d'une constitution démocratique.

Le but de ma présentation était de définir et de défendre une forme de constitutionnalisme démocratique qui soit capable d'éviter cette conséquence paradoxale. Dans le constitutionnalisme démocratique, l'autorité d'interpréter et, possiblement, de renouveler l'interprétation de la constitution revient de façon égale aux trois pouvoirs législatif, exécutif et judiciaire. Les précédentes tentatives pour concevoir un constitutionnalisme "populaire» ou "politique», selon les formules respectives de Larry Kramer et de Richard Bellamy, se sont à raison vu objecter le fait qu'elles réduisent la démocratie au pur gouvernement du nombre: un régime démocratique dont la constitution n'est pas soustraite à la volonté changeante des gouvernants, qui peuvent donc constamment la modifier, n'aurait de constitutionnel que le nom. J'ai tâché de montrer que le constitutionnalisme démocratique échappe à cette critique, et sauve donc la démocratie du pur procéduralisme, en faisant en sorte que les conditions dont la satisfaction est nécessaire pour que l'on puisse parler de démocratie ne puissent qu'être librement déterminées par les membres de la communauté démocratique eux-mêmes. 
Arnaud SOROSINA

Les historicités de Nietzsche

Thèse dirigée par Bertrand Binoche arnaud.sorosina@yahoo.fr

\section{Les figures de l'oubli chez Nietzsche}

L'examen des différents concepts de l'oubli chez Nietzsche permet de rendre compte de son évolution philosophique : en 1873-1874, Nietzsche définit l'oubli du point de vue de sa forme, comme une activité de sélection des données mnésiques à partir de catégories organiques qui sont par définition des «falsifications». L'oubli est pensé comme l'essence du vivant, et défini comme « force plastique » qui ne s'alimente qu'à la source du mythe (wagnérien). Du matériau historique, il n'est pas encore question, sinon pour le mettre sous tutelle de la vie, de telle sorte que l'oubli est défini quant à sa forme, mais non quant à son contenu.

De la métaphysique d'artiste de ses jeunes années bâloises à la «philosophie historique» défendue dans Humain, trop humain (1878), Nietzsche récuse cette conception de l'oubli en congédiant la mythopoïèse wagnérienne. L' " esprit libre » se met en quête, aux côtés de Paul Rée, d'une archéologie de la mémoire. Mais cette fois-ci il semble au contraire que la forme à donner aux contenus historiques demeure introuvable.

C'est seulement avec l'hypothèse de la volonté de puissance qu'une nouvelle conception de la mémoire permet de penser l'oubli par-delà l'antinomie de sa forme et de son contenu. Désormais, l'oubli est caractérisé comme l'expression d'une volonté de puissance capable de maîtriser ce qu'elle a assimilé, c'est-à-dire mémorisé. L'oubli n'est plus un simple cadre transcendantal qui conditionne la réception du donné dans la mémoire, ni au contraire un ensemble de contenus mnésiques abandonnés à l'érosion 
naturelle du temps, mais l'activité de la volonté de puissance qui sélectionne, trie et hiérarchise les évaluations. Ainsi, dans la Généalogie de la morale, le concept synthétique d' «oubli actif» permet de récuser l'opposition entre la mémoire et l'oubli. Il s'agit de congédier la conception statique de la mémoire comme conservatoire des souvenirs. C'est le degré d'activité (ou de passivité - l'opposition n'a plus qu'un sens polaire) d'une volonté de puissance, qui autorise désormais à décrire l'oubli tantôt comme inféodation de certaines traces mnésiques à d'autres (oubli actif propre à la grande santé), tantôt comme recouvrement inertiel des traces (source du ressentiment et des diverses fossilisations donnant naissance aux illusions de la grammaire). Aussi Nietzsche peut-il décrire le processus par lequel la volonté de puissance croît à proportion de sa capacité à oublier : une société est d'autant plus puissante qu'elle est moins contrainte de réprimer sévèrement les crimes, dans la mesure où ceux-ci ne l'affectent pas. Le droit devient moins répressif que dissuasif, et la société tend alors à oublier le tort commis, jusqu'à ce qu'elle soit même capable de gracier le criminel (Généalogie de la Morale, II, 10). Semblablement, l'individu supérieur capable de faire sien l'amor fati est celui qui n'a pas besoin de réprimer, ni même de pardonner les torts qui lui sont faits, tant il est capable d'en inverser la valeur et de faire que ce qui ne le tue pas le rend plus fort. L'oubli ne consiste donc plus à ignorer ou à refouler, mais à digérer, c'est-à-dire : à transformer en force constitutive du Soi ce qui semblait le menacer de l'extérieur. 
Mathilde UNGER

La justification des frontières nationales de la justice sociale

Thèse dirigée par Jean-Fabien Spitz

Mathildeunger@yahoo.fr

\section{L'objection « nationale » de David Miller aux théories cosmopolitiques sur la justice mondiale}

Dans le débat sur les théories de la justice distributive mondiale, les auteurs dits «cosmopolitiques» affirment que parce que les conditions de production des richesses sont devenues mondiales, que le capital comme le travail circulent de plus en plus librement à travers les frontières, il convient d'envisager la répartition des bénéfices produits et la protection des travailleurs à une échelle supranationale, voire mondiale. Ce texte s'intéresse à une objection formulée contre cette ambition " cosmopolitique » donnée à la justice sociale. Elle défend qu'en dépit de la mondialisation économique et commerciale, le lieu de la justice sociale doit, lui, demeurer national.

David Miller a donné à cette idée une élaboration théorique, selon laquelle les principes de justice sociale sont nés et ont été rendus possibles à l'intérieur d'un contexte de solidarité national. Il n'y aurait pas de sens, selon lui, à vouloir sortir les droits et les devoirs sociaux hors des frontières de l'État-nation puisque c'est le contexte national qui a dessiné leur contenu et qui doit donc continuer à délimiter leur domaine d'application. Il défend que l'État-nation constitue à la fois le contexte d'apparition et le lieu de justification des principes de justice sociale, ou plutôt, qu'il en dessine le domaine d'application légitime car il en a été le berceau historique.

Il s'agit d'éclairer l'argument sur l'origine nationale de la justice sociale par la définition que Miller donne de la «nationalité » comme culture publique partagée: elle permettrait de forger la conscience d'appartenir à 
une communauté délimitée et de bâtir un consensus collectif sur la signification des biens sociaux, deux conditions de possibilité de la justice sociale. Cet article vise ensuite à identifier le statut de l'argument historique mobilisé et à examiner sa vulnérabilité potentielle face à des récits concurrents sur les conditions d'émergence de la justice sociale. Si le rôle $\mathrm{du}$ sentiment patriotique glorifié par les deux Guerres mondiales dans l'établissement des États providence est largement attesté, il n'est pas sûr qu'il traduise la solidarité préexistante d'une communauté homogène ni un consensus national sur le sens de la justice.

Le développement de régimes d'assurance locaux organisés par professions permet, par exemple, de relativiser le rôle que l'idée de communauté nationale délimitée aurait eu dans la mise en place de la protection sociale. C'est toutefois les luttes sociales à l'origine de l'acquisition des droits sociaux qui affaiblissent le plus sérieusement la fonction consensuelle qu'aurait pu avoir la culture nationale dans leur apparition.

Comme l'enjeu de l'argument historique reste pour Miller de consolider une thèse normative sur l'applicabilité des principes de justice hors des frontières nationales, il faut alors renverser le raisonnement et se demander si le fait de retrouver, à l'échelle mondiale, certains phénomènes que ces hypothèses alternatives comptent parmi les facteurs déterminants pour expliquer les progrès historiques en matière de justice sociale ne doit pas nous faire réviser les frontières de leur application légitime. 